\title{
Article
}

\section{Medical professionalism: navigating modern challenges}

Cooper-Moss, Nicola, Hooper, Helen, Choong, Kartina Aisha and Chauhan, Umesh

Available at http://clok.uclan.ac.uk/39355/

Cooper-Moss, Nicola, Hooper, Helen, Choong, Kartina Aisha ORCID: 00000001-9407-1771 and Chauhan, Umesh ORCID: 0000-0002-0747-591X (2022) Medical professionalism: navigating modern challenges. InnovAIT, 15 (1). pp. 7-13. ISSN 1755-7380

It is advisable to refer to the publisher's version if you intend to cite from the work. http://dx.doi.org/10.1177/17557380211052669

For more information about UCLan's research in this area go to http://www.uclan.ac.uk/researchgroups/ and search for <name of research Group>.

For information about Research generally at UCLan please go to http://www.uclan.ac.uk/research/

All outputs in CLoK are protected by Intellectual Property Rights law, including Copyright law. Copyright, IPR and Moral Rights for the works on this site are retained by the individual authors and/or other copyright owners. Terms and conditions for use of this material are defined in the policies page.

\section{CLoK}

Central Lancashire online Knowledge www.clok.uclan.ac.uk

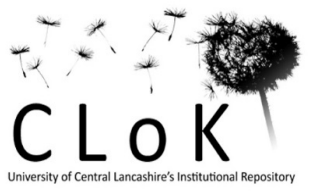




\section{Medical professionalism: navigating modern challenges}

Medical professionalism is an evolving entity, requiring continual development according to shifting societal priorities. The public trust which underpins the medical profession is imperative for maintaining effective partnerships with patients, their families and the wider community. This article provides an overview of what constitutes medical professionalism, including the current protocols and assessments for general practice training. The aim is to improve understanding of the current issues surrounding professionalism in primary care. Fictional case scenarios are used to illustrate modern professional dilemmas and to promote reflection on the complex interacting factors which influence professional practice and clinical decision-making.

\section{Clinical case scenario 1}

Mrs A is a 35-year-old female solicitor, whom you meet for the first time. She has made an appointment to discuss her persistent low back pain, following a diagnosis of mechanical back pain by the musculoskeletal team. She is dissatisfied with this diagnosis and has read extensively about back pain on various websites. She informs you that she consulted an "online doctor" two days ago who suggested that she should be referred for a Magnetic Resonance Imaging (MRI) scan. On explaining your reservations about the MRI scan, Mrs A becomes angry and demands to speak to someone more senior.

\section{Reflective task:}

Consider the following questions relating to this case:

- How do digital technology and access to information impact professionalism?

- What is your emotional reaction to the information presented in this case?

- What are the factors which influence your decision-making in this case?

- What is your standpoint on investigation protocols? Has this changed during different stages of your training and career?

\section{Background and definitions}

The conceptualisation of medical professionalism has developed over thousands of years and continues to receive much research attention today. The Royal College of Physicians (2005) has defined medical professionalism as: "a set of values, behaviours, and relationships that underpins the trust the public has in doctors". At first glance, this definition appears to describe a simple transaction between doctors and society, however, the reality of modern medicine is complicated by shifting societal expectations and multifaceted contextual factors. Some of the 
early principles, such as the morality of medicine, have endured the millennia and others are continually evolving.

Narratives considering the moral obligations of a doctor can be traced back to Hippocrates (460-377 BC) and the writings of Scribonius Largus, a physician in the Roman Empire era (Edelstein, 1943; Hamilton, 1986). The word "profess", first written by Largus in a book of prescriptions, refers to a public commitment or declaration to a set of values. These expectations of public duty and trust remain intrinsic to contemporary definitions. In the United Kingdom (UK), the importance of maintaining high ethical standards among doctors was made explicit in 1858 by the establishment of the regulatory function of the General Council of Medical Education and Registration - the predecessor of the General Medical Council (GMC), and given greater clarity more recently (General Medical Council, 2013).

Early perspectives on medical professionalism were largely based on paternalistic models of care. This has gradually changed from the 18th century onwards, as a result of the bioethical teachings of two physicians: John Gregory and Thomas Percival. The term "patient", originating from the Latin term for passion, was increasingly used to refer to ill persons; thus, signifying that patients are those requiring compassion in a relationship (Rosen, 1992). Monrouxe and Rees (2017) describe principlism as the dominant discourse to which healthcare students are exposed during initial training-principle ethics comprising familiar concepts such as "first do no harm", informed consent, acting fairly and for the benefit of patients. However, person-based virtue ethics, where humanistic traits such as integrity, altruism, accountability and respect further define the doctor-patient relationship and can be seen as influential in decisions made by any doctor. Nonetheless, the inclusion of humanistic facets in modern definitions of professionalism is still debated, and their measurement and assessment remain problematic. This complexity is further highlighted in a multinational interview study by Monrouxe and Rees (2017) who demonstrate how professionalism can be defined and interpreted differently according to professional and cultural contexts, the particular discourse applied and the interaction of practical wisdom with professional identity development.

Bioethicists prioritise virtue ethics in their discourse (Cruess \& Cruess, 2008), where professionalism is seen as the application of a set of personal values rather than a collective responsibility and social contract. They argue that the teaching of professionalism should include the moral basis of medicine while considering our obligations to society and a 
knowledge of self-regulatory processes. In keeping with the more integrated and inclusive approach to the understanding and teaching of professionalism advocated by Cruess and Cruess, the choice of reflective questions in this article facilitates consideration of the interactions of personal, organisational and contextual factors within the example case studies.

\section{Professional capabilities of a general practitioner}

There are many attitudes and behaviours expected of a good doctor, including an ability to justify one's decisions and actions at all times (General Medical Council, 2013). Demonstration of professional capability goes beyond application of sound medical knowledge and skills. Professional capabilities also include values and behaviours required for professional practice. Figure 1 provides an overview of the generic professional capabilities framework, on which the GP training curriculum is based.

\section{(Insert Figure 1 here)}

In their report for the Royal College of Physicians, Tweedie, Hordern and Dacre (2018) describe seven core professional roles of modern doctors, which are particularly pertinent to general practice. These include the doctor functioning as a healer, patient partner, team worker, manager and leader, advocate, learner and teacher and innovator. Readers are encouraged to consider how each of these roles are affected by shifts in societal expectations and changes in medical practice.

\section{Emerging challenges}

Van Mook et al. (2009) describe a new era of medical professionalism based on negotiation and balance between individual and organisational needs, whilst navigating a wide range of professional dilemmas. There are arguably more moral dilemmas associated with the delivery of healthcare now than ever before, with even less time to reflect on them as they occur. Despite many good intentions for person-centred care, the sustainability of healthcare systems relies on prioritisation of efficiency and cost-saving. GPs are therefore faced with situations where ethical principles compete. For example, changing to more cost-effective brands of medication represents a familiar dilemma where principles of utilitarianism and upholding individual patient autonomy are competing priorities in GPs' decision-making. Table 1 provides examples of modern challenges encountered in general practice and the potential impacts on 
patient care. This article explores some of these challenges in further detail. Readers are directed to Monrouxe and Rees (2017) and Tweedie, Hordern and Dacre (2018) for further consideration of challenges not discussed in this short article.

GPs are also required to appreciate changes in the social landscape. For example, there is now increasing recognition that gender identity is a spectrum, with male and female at either end and a range of gender identities in between. Similarly, there is growing support for the recognition of a wider range of sexual orientations, for example, lesbian, gay, bisexual, transgender, queer and questioning (LGBTQQ). The importance of attentiveness is further emphasised by recent social movements, such as the Black Lives Matter campaign. GPs have a key role in fostering equality, inclusivity and diversity within their practice; ensuring that all patients can access healthcare without discrimination or harassment from staff members and other patients. They also need to be aware of the specific health risks and needs of minority communities, and be sensitive to the significance of a safe, welcoming and non-judgmental environment. This includes the use of inclusive language in written communications (NHS Digital Service Manual, 2020).

Another challenge that GPs face is the need to be up-to-date with recent legal developments that affect clinical practice. In the last few years, there have been changes, for instance, to the laws on information disclosure (Montgomery v. Lanarkshire Health Board [2015] UKSC 11), data protection (General Data Protection Regulation 2016/679), organ donation (Organ Donation (Deemed Consent) Act 2019), and children and young persons' consent to puberty blocking medication (Bell v. Tavistock and Portman NHS Foundation Trust [2020] EWHC 3274). More recently, they are also expected to familiarise themselves with the Coronavirus Act 2020 and the various statutory instruments which have been issued relating to the COVID19 pandemic.

Social networking and sharing of information via online platforms, collectively referred to as social media, have also become an integral part of societal interaction, promoting a culture of informality, openness and wide-reaching connections. As these values contrast sharply with traditional professional principles of formality, confidentiality and privacy (George et al., 2013), GPs would need to keep in mind that the standards of communication expected of them on social media are the same as those in other modes of clinical interaction. It is therefore imperative that they follow the Royal College of General Practitioners' (RCGP) Social Media 
Highway Code (2013) and the guidelines issued by the GMC on Doctors' Use of Social Media (2013). Of note is the requirement to uphold patient confidentiality including when sharing workrelated experiences even in professional forums. Additional care to maintain professional boundaries may also be required. In situations where patients contact them about their care or other professional matters via their private (non-professional) profile, GPs are advised to tell patients that they are not able to mix social and professional relationships. Where appropriate, they should consider directing patients to their professional profile. By the same token, they would need to apply their professional judgment and common sense before accepting "friend" requests from patients on social media sites like Facebook. While opinions will differ as to whether such virtual boundaries compromise the partnership and mutuality that bind the modern doctor-patient relationship, obvious issues with confidentiality and privacy may result, thus posing a greater threat to the trust which underpins the medical profession as a whole. GPs must further ensure that the usual requirements to treat colleagues fairly and respectfully extend to online communications. They should therefore refrain from any actions which may be perceived as bullying or harassing, or from making gratuitous, unsubstantiated or unsustainable comments about others on social media.

Recent years have also shown changes to doctors' attitudes and priorities. There are now more female doctors and more GPs working less than full time than ever before. With this comes an increased emphasis on the personal wellbeing of doctors, which some GPs may experience as a conflict between self-interest and a desire to altruistically help others. Jones (2019) highlights the specific tensions building in general practice over recent years, including a culture of "clock-watching" and a restricted number of problems per consultation. In addition to increasing time pressures, there are challenges associated with the expansion of a variety of consultation methods, including video, telephone, email and text messaging.

(Insert Table 1 here)

\section{Professionalism and COVID-19}

The COVID-19 pandemic has led to substantial changes in practice and priorities for people of all backgrounds. Many health professionals have faced unprecedented pressures which have tested their personal attitudes and values surrounding their duty-of-care for patients and its 
limits. Shi and Jiao (2020) suggest that the COVID-19 pandemic has presented a novel opportunity to model and learn about medical professionalism, as well as rebuilding public confidence and cohesion.

A vast majority of patient care during the COVID-19 pandemic have been delivered via telephone and online consultations in order to minimise the spread of the novel coronavirus (Greenhalgh et al., 2020). Practices have therefore had to face the challenge of adapting to remote working without sufficient time nor opportunity to reflect on their implications for quality of care. However, since any delay in or suspension of service would be far more detrimental to patients, GPs have demonstrated adaptability and continuing commitment to patient welfare by providing ongoing care during the pandemic (Thornton, 2020). Indeed, those who still provide face-to-face consultations for urgent cases, including visiting housebound patients, have even had to balance acceptable personal risks with public duty (Lacobucci, 2020).

Further, there is the emergence of an increasing number of professional guidelines relating to medical care during the pandemic. These are issued by organisations like the RCGP, the British Medical Association (BMA), the GMC, the National Institute for Health and Care Excellence (NICE), NHS England and Public Health England. Some of these guidelines are regularly updated, which has reinforced the importance of keeping up-to-date. GPs are similarly expected to play a key role in assuring patients about the safety of new aspects of their medical care, such as COVID-19 vaccinations, whilst acknowledging medical uncertainty and recognising limitations in their own expertise. Together with the influx of research findings on COVID-19, the various guidelines and advice have the potential to create a heady mix of information overload, stressful workload and changeable patient expectations.

Case scenario 2 presents a fictional professional dilemma during the COVID-19 pandemic. The learning can be applied to similar situations involving medical uncertainty.

\section{Case scenario 2}


Mr B, a 55-year-old African-Caribbean male patient has requested a telephone consultation to discuss his invitation for COVID-19 vaccination. You have developed a good relationship with him over the last 12 months following his diagnosis of essential hypertension. He is otherwise well and is the main carer for his wife who has Multiple Sclerosis. He is concerned about potential serious side effects, such as blood clots, which he has read about in the newspaper. Additionally, some of his friends on Facebook have reported flu-like symptoms for several days after the vaccine and he is worried about not being able to care for his wife. He asks you what he should do.

\section{Reflective task:}

Consider the following questions relating to this case:

- What is your role as a GP in supporting this patient to decide?

- How does the uncertainty in this case affect your professional advice?

- How do you feel about providing reassurance to patients when research is still being undertaken, not all trials are complete and knowledge is developing rapidly?

- Are there contextual factors in this case which may influence your approach?

- How might your pre-conceptions and expectations of this patient influence your stance?

\section{Assessment and protocols in general practice}

Professionalism is an arduous concept to quantify and there is limited evidence to support the utility of individual measures. Hence, a combination of assessments is required to effectively encompass the multidimensions of professionalism (Lynch et al., 2004; Li et al., 2017). This section describes some of the main assessments and protocols for professionalism in general practice training and beyond. It is important that GPs have an understanding of these processes in order to optimise both their own learning and professional development and the teaching and feedback they provide to others on professionalism.

Professionalism may be best assessed by direct observation, however, this method is not always possible due to time and cost implications. Furthermore, observable behaviours may 
not always correlate with underlying attitudes in singular situations and it is debatable whether attitudes are distinguishable through examination questions (O'Shaughnessy and Joyce, 2015). The case scenarios in this article were designed to encourage consideration of feelings, values and standpoints which may constitute potential influences on decisions made in specific situations and, in this respect, self-awareness is encouraged (Papanikitas, 2017).

Lynch et al. (2004) noted a trend to move away from summative assessment formats and to evaluate professionalism more formatively over time. Formative assessment provides an opportunity for detailed narrative feedback which supports longitudinal development and has an increasing role in GP training. The GP training and appraisal portfolios include a range of assessment formats which demonstrate a variety of professional qualities (Table 2). This could be considered an example of the more rounded assessment of professionalism recommended by Shaughnessy and Joyce (2015). Appraisals and annual training reviews facilitate reflective learning on a wide spectrum of evidence, including learning events, feedback from patients and colleagues, and quality improvement activities (Royal College of General Practitioners, 2018). Formative assessments such as multi-source feedback and patient questionnaires may also highlight professionalism concerns which are otherwise overlooked in self-assessments.

Beyond training, professional capability is demonstrated through continuing professional development, appraisal and revalidation. The revalidation process includes an annual appraisal of supporting evidence and a five-yearly revalidation assessment by a Responsible Officer (RO). Fitness to practise is demonstrated by having insight into health or conduct issues that may impact personal performance or put patients at risk. GPs should anticipate and manage the factors which influence their day-to-day performance at work, home and wider environments, including the virtual environment.

\section{(Insert Table 2 here)}

\section{Opportunities for professionalism development}

The assessment processes outlined in Table 2 all provide opportunities for professionalism development. In addition, the multitude of professional dilemmas seen in general practice (Table 1) provide opportunities for a combination of experiential learning, informal feedback and critical reflection. Reflective practice is considered in more detail here, however, readers 
are also referred to the various scenarios and prompts outlined in "Advancing medical professionalism" for further developmental strategies (Tweedie, Hordern and Dacre, 2018).

\section{Reflective practice}

Hilton (2004) argues that a combination of high levels of ethical functioning and reflective judgement are required to navigate modern problems of society which are frequently complex and conflicting. However, as with professionalism, reflexivity is an abstract concept with many varying definitions and recommended approaches. It is generally assumed, however, that critical reflection has the potential to contribute to improvements in professional practice (Ghaye and Lillyman, 2010). Papanikitas (2017) suggests that reflection supports selfawareness and encourages consideration of how our individual goals, beliefs and values align with societal expectations and the systems in which we work. Another school of thought is that reflection pulls together, and helps us to make sense of, our previous knowledge and ways of thinking - a way of looking at our practice from different angles, or perspectives (Jasper et al., 2013). The central tenet of all approaches to reflective practice is that we must be prepared to learn continually from our experiences in order to improve and develop knowledge, skills and attitudes. This includes reflection on the professional behaviours displayed by clinical rolemodels.

The GMC have also published guidance on reflective practice, which addresses the rising concerns regarding openly reflecting on mistakes involving patient care (General Medical Council, 2018). Such concerns have resulted from high profile cases of clinical negligence, such as the Bawa-Garba case, and remain a real issue for doctors today. Nevertheless, reflective practice and self-awareness remain central components of professionalism, and all doctors should be afforded time and support to reflect on both positive and negative experiences. GPs are encouraged to reflect both individually and in teams, focussing on learning, developmental outcomes and future plans. Written reflections should be anonymised where possible and lengthy descriptions of the case are usually unnecessary for the purposes of reflective learning cycles. The GMC guidance also provides suggestions for structured approaches to reflection, which some individuals may find helpful.

\section{Learning from mistakes}


Acknowledging and learning from mistakes demonstrate abstractions of professionalism, such as accountability, integrity and self-regulation, which are otherwise difficult to demonstrate in simulated situations. Quality improvement activities such as Significant Event Analysis (SEA) provide an opportunity for structured team-based learning from a wide range of clinical and non-clinical experiences, including those involving adverse incidents and diagnostic delays (Cooper-Moss et al., 2020).

It is also argued that true professionalism is demonstrated when mistakes are made (Medical Protection Society, 2017). In the case of FB v. Princess Alexandra Hospital NHS Trust [2017] EWCA Civ 334 where a junior doctor was found negligent for inadequate history-taking, Lord Justice Jackson ended his judgement with a pertinent statement: "Doctors, however, are human. Even good and conscientious doctors may, from time to time, fall short. That is not a reason to lose heart or (even worse) to abandon medical practice. Those who have learnt from past mistakes often have even more to offer".

\section{Conclusions}

Professionalism is an active and effortful developmental process and, as the literature suggests, replete with evolving definitions and indicators that reflect wider healthcare system priorities. Providing the high-quality care which is expected by society has become increasingly challenging, as there is often a disparity between patient expectations and organisational demands. GPs and trainees are encouraged to utilise the broad range of assessment methods within their portfolios as a means of professional development, as well as regularly reflecting on their own experiences of professionalism, so as to better understand the influential intersections of their day-to-day decision-making and fitness to practise. Further research is required into more rounded measures of assessment and into the contextual factors influencing both attitudinal and behavioural changes. 


\section{Key points}

- Professionalism is a complex and evolving concept which is socially determined.

- Professional capability encompasses more than just knowledge and skills.

- The development of professional capabilities requires life-long engagement in a combination of self-directed learning, formative feedback and critical reflection.

- Reflective practice promotes self-awareness, which is required for navigating the rapidly changing environment which is healthcare.

- Modern professional dilemmas, including the challenges associated with the COVID-19 pandemic, provide rich learning opportunities for professional development and immersion teaching on professionalism.

\section{References and further information}

Cooper-Moss N, Smith N and Chauhan U (2020) The importance of significant event analysis. InnovAiT 13(10): 613-617. DOI: 10.1177/1755738020941231.

Cruess SR and Cruess RL (2008) Understanding medical professionalism: a plea for an inclusive and integrated approach. Medical Education 42(8): 755-757. DOI: 10.1111/j.1365-2923.2008.03134.x

Department of Health (2012) NHS Constitution for England. Available at: https://www.gov.uk/government/publications/the-nhs-constitution-for-england (accessed 16 May 2021).

Edelstein L (1943) The Hippocratic Oath: Text, Translation and Interpretation. Baltimore: Johns Hopkins Press.

General Medical Council (2013) Good medical practice. Available at: https://www.gmcuk.org/ethical-guidance/ethical-guidance-for-doctors/good-medical-practice (accessed 16 May 2021).

General Medical Council (2017) Generic Professional Capabilities Framework. Available at: https://www.gmc-uk.org/-/media/documents/generic-professional-capabilitiesframework--0817_pdf-70417127.pdf (accessed 16 May 2021).

General Medical Council (2018) The reflective practitioner - guidance for doctors and medical students. Available at: https://www.gmc-uk.org/education/standards-guidance-and- 
curricula/guidance/reflective-practice/the-reflective-practitioner---guidance-for-doctorsand-medical-students (accessed 16 May 2021).

George DR, Rovniak LS and Kraschnewski JL (2013) Dangers and opportunities for social media in medicine. Clinical Obstetrics and Gynecology 56(3): 453-462. DOI: 10.1097/GRF.0b013e318297dc38.

Ghaye T and Lillyman S (2010) Reflection: Principles and Practices for Healthcare Professionals. London: Quay Books.

Greenhalgh T, Koh GC and Car J (2020) Covid-19: a remote assessment in primary care. The BMJ 368. DOI: 10.1136/bmj.m1182.

Hamilton JS (1986) Scribonius Largus on the medical profession. Bulletin of the History of Medicine 60(2): 209-16.

Hilton S (2004) Medical professionalism: how can we encourage it in our students? The Clinical Teacher 1(2): 69-73. DOI: 10.1111/j.1743-498X.2004.00032.x.

lacobucci G (2020) Doctors still at "considerable risk" from lack of PPE, BMA warns. The BMJ 368. DOI: $10.1136 / \mathrm{bmj} . \mathrm{m} 1316$.

Jasper M, Rosser M and Mooney G (2013) Professional Development, Reflection and Decision-making in Nursing and Healthcare. Swansea, UK: Wiley-Blackwell.

Jones R (2019) In place of fear. British Journal of General Practice 69(685): 371. DOI: 10.3399/bjgp19X704669.

Li H, Ding N, Zhang Y et al. (2017) Assessing medical professionalism: a systematic review of instruments and their measurement properties. PLoS ONE 12(5). DOI: 10.1371/journal.pone.0177321.

Lynch DC, Surdyk PM and Eiser AR (2004) Assessing professionalism: a review of the literature. Medical teacher 26(4): 366-373. DOI: 10.1080/01421590410001696434.

Medical Protection Society (2017) Chapter 1: Medical Professionalism - What do we mean? Available at: https://www.medicalprotection.org/uk/articles/chapter-1-medicalprofessionalism-what-do-we-mean (accessed 19 May 2021).

Monrouxe LV and Rees CE (2017) Healthcare Professionalism: Improving Practice through Reflections on Workplace Dilemmas. Chichester, UK: John Wiley \& Sons. 
NHS Digital Service Manual (2020) Inclusive language. Available at: https://servicemanual.nhs.uk/content/inclusive-language (accessed 16 May 2021).

NHS England (2017) Involving people in their own health and care: Statutory guidance for clinical commissioning groups and NHS England. Available at: www.england.nhs.uk/wp-content/uploads/2017/04/ppp-involving-people-health-careguidance.pdf (accessed 16 May 2021).

O'Shaughnessy, SM and Joyce P (2015) Summative and Formative Assessment in Medicine: The Experience of an Anaesthesia Trainee. International Journal of Higher Education 4(2): 198-206. DOI:10.5430/ijhe.v4n2p198.

Papanikitas A (2017) Self-awareness and professionalism. InnovAiT 10(8): 452-457. DOI: $10.1177 / 1755738017710962$.

Rosen DH (1992) Inborn basis for the healing doctor-patient relationship. Pharos Alpha Omega Alpha Honor Medical Society 55(4):17-21.

RCGP (2018) RCGP Guide to supporting information for appraisal and revalidation. Available at: https://www.rcgp.org.uk/-/media/Files/Revalidation-and-CPD/2018/RCGP-guide-tosupporting-information-2018.ashx?la=en (accessed 16 May 2021).

Royal College of Physicians (2005) Doctors in society: medical professionalism in a changing world. Report of a working party of the Royal College of Physicians of London. London: RCP. Available at: https://www.rcplondon.ac.uk/file/11741/download?token=p-IUq0QX (accessed 16 May 2021).

Shi W and Jiao $Y$ (2020) The COVID-19 pandemic: a live class on medical professionalism. QJM: An International Journal of Medicine. DOI: 10,1093/qjmed/hcaa157.

Thornton J (2020) Covid-19: how coronavirus will change the face of general practice forever. The BMJ 368. DOI: $10.1136 / \mathrm{bmj} . \mathrm{m} 1279$.

Tweedie J, Hordern J and Dacre J (2018) Advancing medical professionalism. Royal College of Physicians.

Van Mook WN, De Grave WS, Wass V et al. (2009) Professionalism: evolution of the concept. European Journal of Internal Medicine 20(4): 81-84. DOI: 10.1016/j.ejim.2008.10.005. 
Figure 1: Overview of the generic professional capabilities framework for doctors

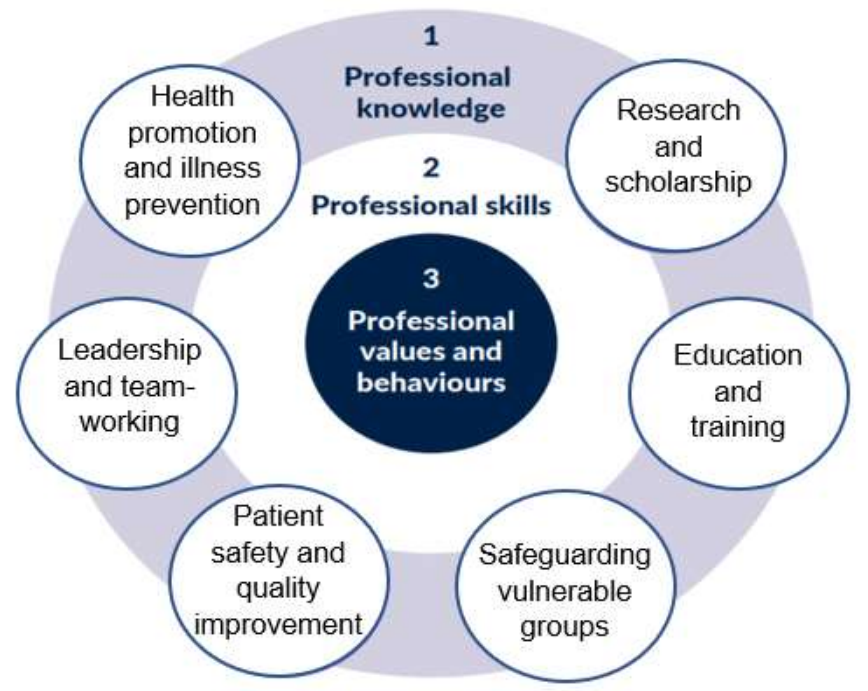

General Medical Council (2017: 5) 
Table 1: Modern professional dilemmas in general practice

\begin{tabular}{|c|c|}
\hline Professional dilemma & Potential impact on patient care \\
\hline $\begin{array}{l}\text { Rationing, consumerism and concern } \\
\text { for profit }\end{array}$ & $\begin{array}{l}\text { Patient dissatisfaction, public distrust, conflicts with } \\
\text { autonomy, beneficence, non-maleficence and justice }\end{array}$ \\
\hline "Expert" and health literate patients & $\begin{array}{l}\text { Shifts in doctor-patient relationship dynamics, conflicts } \\
\text { with organisational priorities }\end{array}$ \\
\hline $\begin{array}{l}\text { Online social networking and } \\
\text { information sharing }\end{array}$ & $\begin{array}{l}\text { Unclear boundaries, breaches in confidentiality, public } \\
\text { scrutiny, finding useful resources more easily }\end{array}$ \\
\hline Media publicity and commercialism & Public generalisations, blame and litigation culture \\
\hline $\begin{array}{l}\text { Increase in digital technology and } \\
\text { access of information }\end{array}$ & $\begin{array}{l}\text { Concerns for confidentiality, consent and data } \\
\text { protection, variable communication and technical } \\
\text { proficiency among doctors and multidisciplinary teams }\end{array}$ \\
\hline Change in consultation methods & $\begin{array}{l}\text { Patient and doctor dissatisfaction, remote consultation } \\
\text { skills development, variable communication }\end{array}$ \\
\hline $\begin{array}{l}\text { Rapid increase in clinical } \\
\text { guidelines and medical laws }\end{array}$ & $\begin{array}{l}\text { Clinical autonomy versus standardised (measurable) } \\
\text { care, variable implementation and compliance }\end{array}$ \\
\hline $\begin{array}{l}\text { Changes in social, cultural and } \\
\text { environmental landscape }\end{array}$ & $\begin{array}{l}\text { Continual self-regulation and adaptation of } \\
\text { approaches to maintain respect and justice for } \\
\text { patients }\end{array}$ \\
\hline $\begin{array}{l}\text { Changes in doctors' attitudes and } \\
\text { priorities }\end{array}$ & $\begin{array}{l}\text { More GPs working less than full time, increased } \\
\text { emphasis on personal wellbeing, feminisation of } \\
\text { medicine, conflicts with altruism and self-preservation }\end{array}$ \\
\hline $\begin{array}{l}\text { Recognising and reporting } \\
\text { unprofessional behaviour in } \\
\text { others }\end{array}$ & Maintenance of public trust and patient safety \\
\hline
\end{tabular}


Table 2: Assessment of professionalism in general practice

\begin{tabular}{|l|l|}
\hline $\begin{array}{l}\text { Training / Appraisal } \\
\text { portfolio item }\end{array}$ & Aspect of professionalism \\
\hline $\begin{array}{l}\text { Continuing professional } \\
\text { development }\end{array}$ & - Keeping up-to-date with clinical knowledge, skills and changes in \\
policies, laws and guidelines affecting clinical care
\end{tabular}

